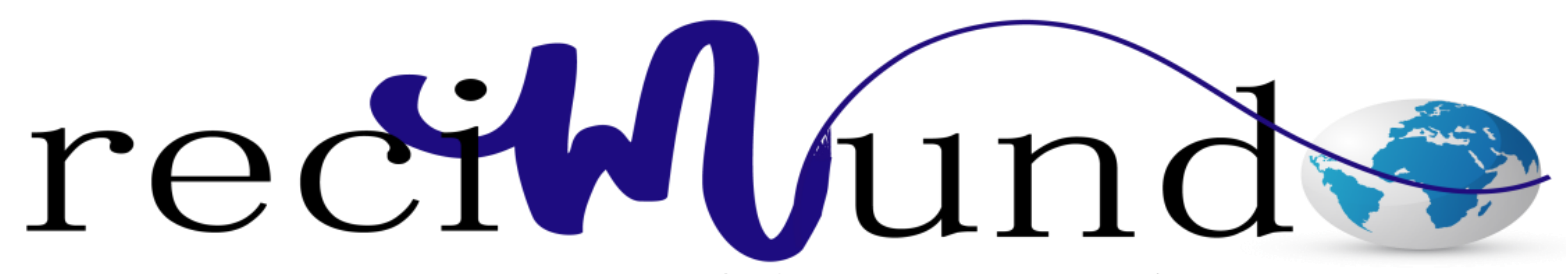

Revista Cientifica Mundo de la Investigación y el Conocimiento

Marcos Alfredo Romero Pérez ${ }^{\mathrm{a}}$; Carlos Bruno Guerrero ${ }^{\mathrm{b}}$;

Nina Paulette Arteaga Vélez ${ }^{\mathrm{c}}$

La capnografía: estudio fundamental en el monitoreo del paciente anestesiado

Capnography: fundamental study in the monitoring of the anesthetized patient

Revista Científica Mundo de la Investigación y el Conocimiento. Vol. 3 núm.3, septiembre, ISSN: 2588-073X, 2019, pp. 82-95

DOI: $10.26820 /$ recimundo/3.(3).septiembre.2019.82-95

URL: http://recimundo.com/index.php/es/article/view/512

Código UNESCO: 3205 Medicina Interna

Tipo de Investigación: Artículo de Revisión

Editorial Saberes del Conocimiento

Recibido: 15/05/2019

Aceptado: 23/06/2019

Publicado: 30/09/2019

Correspondencia: marcosromero1988@gmail.com

a. Médico; Investigador Independiente; Guayaquil, Ecuador; marcosromero1988@ gmail.com

b. Médico; Investigador Independiente; Guayaquil, Ecuador; drcarlosguerrero@ hotmail.es

c. Médico; Investigador Independiente; Guayaquil, Ecuador. 


\section{La capnografía: estudio fundamental en el monitoreo del paciente anestesiado \\ Vol. 3, núm. 3., (2019) \\ Marcos Alfredo Romero Pérez; Carlos Brunos Guerrero; Nina Paulette Arteaga Vélez}

\section{RESUMEN}

La capnografía es un recurso que permite optimizar la seguridad del paciente anestesiado y representa una base de vital importancia en la filosofía de trabajo de cuidados anestésicos monitorizados. El objetivo principal del presente estudio consiste en revisar y plasmar el uso de la capnografía como elemento fundamental en la monitorización de pacientes bajo anestesia, resaltando su importancia y exponiendo algunos aspectos generales de este tipo de estudio tales como: concepto y fases del capnograma, a los fines de proporcionar una mejor comprensión del tema. El diseño de investigación que se llevó a cabo es de tipo documental o bibliográfico. La capnografía permite confirmar con la detección de $\mathrm{CO} 2$, la intubación endotraqueal y asegurar que la conexión entre el circuito anestésico y la vía respiratoria esté patente, evaluar la ventilación/perfusión pulmonar, así como la detección de alteraciones en el gasto cardíaco, cambios en la producción o en la eliminación del bióxido de carbono, embolismo pulmonar, entre otros. En conclusión, la capnografía constituye el estándar de oro de la seguridad del paciente bajo anestesia y se encuentra en un nivel altamente recomendado en todas las guías de prácticas clínicas para procedimientos que involucren anestésicos.

Palabras Claves: Capnografía; Estudio; Monitoreo; Anestesia; Paciente. 


\title{
La capnografía: estudio fundamental en el monitoreo del paciente anestesiado
}

Vol. 3, núm. 3., (2019)

Marcos Alfredo Romero Pérez; Carlos Brunos Guerrero; Nina Paulette Arteaga Vélez

\begin{abstract}
Capnography is a resource that optimizes the safety of the anesthetized patient and represents a vital basis in the work philosophy of monitored anesthetic care. The main objective of this study is to review and capture the use of capnography as a fundamental element in the monitoring of patients under anesthesia, highlighting its importance and exposing some general aspects of this type of study such as: concept and phases of the capnogram, to The purpose of providing a better understanding of the subject. The research design that was carried out is documentary or bibliographic. Capnography allows confirming with $\mathrm{CO} 2$ detection, endotracheal intubation and ensuring that the connection between the anesthetic circuit and the airway is patent, assess pulmonary ventilation / perfusion, as well as the detection of changes in cardiac output, changes in the production or in the elimination of carbon dioxide, pulmonary embolism, among others. In conclusion, capnography constitutes the gold standard of patient safety under anesthesia and is at a highly recommended level in all clinical practice guidelines for procedures involving anesthetics.
\end{abstract}

Key Words: Capnography; Study; Monitoring; Anesthesia; Patient. 


\section{La capnografía: estudio fundamental en el monitoreo del paciente anestesiado \\ Vol. 3, núm. 3., (2019) \\ Marcos Alfredo Romero Pérez; Carlos Brunos Guerrero; Nina Paulette Arteaga Vélez}

\section{Introducción.}

Según la Organización Mundial de la Salud (2015) unos 234 millones de intervenciones quirúrgicas, se realizan cada año a nivel mundial, con la finalidad de corregir múltiples afecciones en pacientes de diferentes edades. Entre estas afecciones se destacan: "el parto obstruido, las anomalías congénitas, la diabetes, el cáncer, las enfermedades cardiovasculares, las hernias, las cataratas o las lesiones debidas a accidentes de tráfico, quemaduras y caídas, entre otras”. (p. 3)

La cirugía es un procedimiento que salva vidas en todo el mundo, no obstante, se encuentra asociada a una serie de complicaciones, muchas de las cuales podrían ser susceptibles a prevención. Uno de los mayores riesgos de las cirugías se relaciona con el uso de la anestesia.

La anestesia consiste en la pérdida de sensibilidad y conciencia ocasionada por la administración de medicamentos u otras sustancias (anestésicos). Es utilizada comúnmente durante una operación u otros procedimientos con la finalidad de evitar que el paciente sienta algún tipo de dolor. Se puede clasificar principalmente el tres tipos: la anestesia local que ocasiona pérdida de sensibilidad en un área pequeña del cuerpo del paciente, la anestesia regional, que actúa en una parte del cuerpo (brazo o pierna) y la anestesia general responsable de la pérdida completa de la sensibilidad y la conciencia del paciente. (Instituto Nacional del Cáncer de los Estados Unidos, 2019)

Las guías prácticas o normativas para asistir los procedimientos de anestesiología constituyen una herramienta valiosa en la planificación, puesta en práctica y control de la administración de estos medicamentos. Cada vez más los hospitales, las asociaciones de médicos 


\section{La capnografía: estudio fundamental en el monitoreo del paciente anestesiado}

Vol. 3, núm. 3., (2019)

Marcos Alfredo Romero Pérez; Carlos Brunos Guerrero; Nina Paulette Arteaga Vélez

y los gobiernos se abocan a la temática del mantenimiento de la calidad y seguridad de los pacientes sometidos a la anestesia. El monitoreo del paciente bajo anestesia, se encuentra altamente recomendado en las guías clínicas que regulan esta práctica, es decir, representa un estándar mandatorio que se debe priorizar.

Para Navarret (2011) "la capnografía es el estudio de la forma o diseño de las concentraciones cambiantes de CO2 en el aire espirado”. Este estudio proporciona información de manera rápida y detalladamente, acerca de cada ciclo respiratorio. En la actualidad es vista como uno de los componentes más útiles e importantes de la monitorización de la ventilación.

La capnografía en paciente anestesiado, es un recurso que permite optimizar la seguridad del paciente y representa una base de vital importancia en la filosofía de trabajo de cuidados anestésicos monitorizados.

Hernández Cortez (2017) refiere que “antes de la llegada de la capnografía, la mortalidad por anestesia se encontraba en 1:2500 a 1:5000 pacientes, actualmente se estima 1: por cada 10 000 pacientes". (p. 1) Dicha relación enmarca el avance significativo del uso de esta herramienta de prevención de complicaciones y mortalidad del paciente que debe ser sometido a la anestesia.

El objetivo principal del presente estudio consiste en revisar y plasmar el uso de la capnografía como elemento fundamental en la monitorización de pacientes bajo anestesia, resaltando su importancia y exponiendo algunos aspectos generales de este tipo de estudio tales como: concepto y fases del capnograma, a los fines de proporcionar una mejor comprensión del tema. 


\section{La capnografía: estudio fundamental en el monitoreo del paciente anestesiado \\ Vol. 3, núm. 3., (2019) \\ Marcos Alfredo Romero Pérez; Carlos Brunos Guerrero; Nina Paulette Arteaga Vélez}

\section{Materiales y métodos.}

Para el desarrollo del presente trabajo de investigación se usaron computadores personales con conexión a internet, con el propósito de exponer acerca de la capnografía como elemento fundamental de la monitorización del paciente anestesiado, en virtud de lo cual, el mismo se enmarca en un diseño de investigación documental o bibliográfica.

Dicho estudio se limita a una búsqueda y revisión sistemática de material bibliográfico accesible mediante el uso de diversas bases de datos, entre las que destacaron: MedlinePlus, PubMed, Biblioteca Virtual de la Salud (BVS), SciELO, Dialnet y ELSEVIER, entre otras; mediante las cuales se sintetizó la mejor evidencia de la que se disponía.

Se realizó una búsqueda aleatoria y consecutiva, usando las expresiones "capnografía”, “capnografía y monitoreo"; "capnografía y monitoreo del paciente bajo anestesia" y "importancia de la capnografía", lo que aproximadamente resultó en más de un centenar de registros bibliográficos que se filtraron bajo los criterios de idioma español, relevancia, correlación temática y fecha de publicación en los últimos diez años.

Por último, se le da la correspondiente lectura crítica y análisis de toda esa evidencia científica, lo que resultó consecutiva y consensuadamente en el contenido del presente trabajo.

\section{Resultados.}

Concepto de Capnografía

Pérez de Mendiguren (2017) define a la capnografía como "la monitorización continua y no invasiva de la presión parcial de dióxido de carbono exhalado por el paciente a lo largo del 


\section{La capnografía: estudio fundamental en el monitoreo del paciente anestesiado}

Vol. 3, núm. 3., (2019)

Marcos Alfredo Romero Pérez; Carlos Brunos Guerrero; Nina Paulette Arteaga Vélez

tiempo". Esto es, que la capnografía permite monitorizar la ventilación del paciente, proceso que depende de otros dos que se presentan con anterioridad, el metabolismo, donde se produce el $\mathrm{CO} 2$, y la perfusión que transporta el $\mathrm{CO} 2$ hasta los pulmones, el cual posteriormente es expulsado al exterior. En conclusión, la medición del CO2 exhalado presenta estos tres procesos fundamentales, cuya alteración originará variaciones en los valores de la capnografía. (p. 1)

Respecto a los tipos de medición en la capnografía Barrado, Barroso, Patón, \& Sánchez (2013) destacan que el CO2 exhalado puede ser medidos de dos formas diferentes: como volumen al cual se le denomina capnografía volumétrica y es comúnmente utilizada en pacientes intubados en unidades de terapia intensiva o en quirófanos. La otra forma de medición se denomina capnograma temporal, se trata de la medición de la presión parcial del gas respecto a una línea de tiempo. (p. 16)

Fases de un capnograma

Fase I

Esta fase corresponde al "período comprendido entre el final de la inspiración y el inicio de la espiración siguiente, cuando comienza la ventilación del espacio muerto formado por la vía aérea superior y parte del árbol bronquial que no tienen capacidad para intercambiar gases". Dicho volumen de aire se encuentra casi en su totalidad libre de CO2, lo que lo hace similar al del aire atmosférico. (Barrado et. al, 2013, p. 17) 


\section{La capnografía: estudio fundamental en el monitoreo del paciente anestesiado}

Vol. 3, núm. 3., (2019)

Marcos Alfredo Romero Pérez; Carlos Brunos Guerrero; Nina Paulette Arteaga Vélez

\section{Fase II}

Para Martínez (2012) esta fase está constituida por una "rápida subida de CO2 al inicio de la espiración por la eliminación de CO2 del espacio muerto mezclado con CO2 alveolar”. (p. S19)

La fase III

Con relación a esta fase Ortega (2013) refiere que "es la meseta alveolar que representa la evolución de bióxido de carbono en los alveolos; si esta PCO2 es pareja en todos los alveolos como la meseta alveolar será perfectamente plana”. Es en esta etapa donde verdaderamente se presentan las alteraciones de la relación ventilación/perfusión. (p. S101)

Fase IV

En esta fase es donde comienza la fase inspiratoria, se caracteriza por un decrecimiento veloz de la presión parcial de CO2 hasta llegar y quedarse en cero. "Los cambios en la forma o en la altura de la línea gráfica del capnograma pueden indicar alteraciones en la función respiratoria, la perfusión, el metabolismo del paciente o fallos en el equipo". Por tal razón, es fundamental comprender todas las fases de un capnograma normal a los fines de poder detectar alteraciones en el mismo, por ende, servir de base para indagar acerca de la causa antes de que se presenten complicaciones en el paciente. (Pérez de Mendiguren, 2017, p. 27,28)

Importancia de la capnografía en el área de anestesiología 


\section{La capnografía: estudio fundamental en el monitoreo del paciente anestesiado}

Vol. 3, núm. 3., (2019)

Marcos Alfredo Romero Pérez; Carlos Brunos Guerrero; Nina Paulette Arteaga Vélez

Son múltiples los beneficios del uso de la capnografía en el área médica, constituyéndose hoy por hoy en una herramienta obligatoria en todas las guías de práctica clínica alrededor del mundo, representando una de las bases fundamentales en el ejercicio de las prácticas de cirugía seguras.

La aplicación del monitoreo mediante este estudio ha permitido reducir ampliamente las probabilidades de que se presenten complicaciones asociadas al uso de la anestesia y el estado del paciente sometido a cirugía, además de reducir las tasas de mortalidad asociadas.

Morales Carbonell (2014) destaca que la capnografía se viene usando desde hace más de 30 años con fines de monitorización del paciente intubado en el quirófano y es en esta área donde se ha convertido en el estándar de atención. Los avances tecnológicos han permitido desarrollar capnógrafos portátiles, cuya facilidad de uso ofrece lecturas precisas, usados en pacientes intubados o bien, con ventilación espontánea. Estos nuevos aparatos permiten valorar de forma continua y, cada vez, menos invasiva el metabolismo, la perfusión y la ventilación de los pacientes en tiempo real. (p. 4)

Es importante destacar que la evolución de la capnografía involucra al capnógrafo, el cual en la actualidad es común encontrar asociado a otros equipos que colaboran a la monitorización del paciente bajo anestesia o paciente crítico. Asimismo, se han desarrollado hardware y software que permiten sintetizar toda la información que se registra por medio de estos aparatos e interactuar con esta para la toma de decisiones y la mejor seguridad del paciente. Lo más actualizado en esta materia incluye estudios de monitores inalámbricos, mucho más cómodos, no 


\section{La capnografía: estudio fundamental en el monitoreo del paciente anestesiado}

Vol. 3, núm. 3., (2019)

Marcos Alfredo Romero Pérez; Carlos Brunos Guerrero; Nina Paulette Arteaga Vélez

obstante, su valoración y requerimientos de internet los hacen inaccesibles para muchos países del mundo.

Al respecto, Dell'Osa \& Bernia (2014) definen al monitor multiparamétrico como aquel equipo destinado a la visualización y adquisición conjunta de múltiples parámetros biológicos, entre los que se destacan el electrocardiograma (ECG), oxígeno en sangre (SpO2), presión sanguínea, y capnografía (CO2). Actualmente, existen diversos modelos con diferentes grados de complejidad, cantidad de señales, exactitud y posibilidad de conexión a las centrales de monitoreo, que consisten estas últimas en una computadora con un hardware y un software, donde se puede visualizar la información como ondas temporales y/o valores numéricos. Asimismo, el operador podrá ampliar datos, modificar y/o silenciar alarmas, ver registros anteriores en tiempo, entre otras funciones, que le permitirán de manera más práctica y precisa tomar decisiones en caso de que se presenten variaciones en los valores normales. (p. 1,2)

Para Álvarez \& Ochoa (2013) la capnografía es una medida del metabolismo celular, que durante los procedimientos que requieren anestesia permite: confirmar con la detección de $\mathrm{CO}$, la intubación endotraqueal y asegurar que la conexión entre el circuito anestésico y la vía respiratoria esté patente. Evalúa la ventilación/perfusión pulmonar ya que representa un índice cualitativo y cuantitativo de la ventilación y la perfusión alveolar. Por último, la observación de las variaciones en el valor del $\mathrm{CO} 2$, así como en la morfología del capnograma, son de gran utilidad en la detección de alteraciones en el gasto cardíaco, cambios en la producción o en la eliminación del bióxido de carbono, embolismo pulmonar, entre otros. (p. 95) 


\section{La capnografía: estudio fundamental en el monitoreo del paciente anestesiado}

Vol. 3, núm. 3., (2019)

Marcos Alfredo Romero Pérez; Carlos Brunos Guerrero; Nina Paulette Arteaga Vélez

Otros de los beneficios que se producen con el empleo de la capnografía durante el monitoreo anestésico es la detección temprana de la depresión respiratoria (apnea), hipoventilación y/o desaturación. El Instituto Aragonés de ciencias de la salud describe que "la monitorización de $\mathrm{CO} 2$ mediante capnografía debe ser obligada en toda cirugía, especialmente en la laparoscópica, ya que cualquier variación en la curva del CO2 tele-espiratorio puede ser signo de complicación intraoperatoria, entre ellas la depresión respiratoria". En consecuencia, este procedimiento posee un nivel de evidencia alto y un grado de recomendación fuerte en la escala GRADE (Grading of Recommendations, Assessment, Development and Evaluation). (Pérez de Mendiguren, 2017, p. 3)

Durante la anestesia, es fundamental, el monitoreo sobre la vía aérea y de ventilación. Al respecto, el Checklist para la cirugía segura de la Organización Mundial de la Salud de 2009, citado por Mille, Aréchiga, \& Portela (2011) recomienda "verificar la correcta colocación del tubo endotraqueal y medir su buen funcionamiento con la visualización de la curva de bióxido de carbono expirado". En los casos de ventilación mecánica es recomendable contar con una alarma que informe de la eventual desconexión del paciente ha dicho sistema. Es sugerida la medición continua del volumen de gas inspirado y espirado. (p. S85)

Durante la administración de agentes anestésicos la ventilación del paciente se deprime, es por esta razón que resulta fundamental la monitorización de la ventilación que este requiere durante el procedimiento quirúrgico. En este orden de ideas, Romero, Guerrero, \& Arteaga (2018) resaltan también la importancia de la capnografía al determinar las necesidades de ventilación correctos durante la ventilación controlada. Por otra parte, “en los circuitos de nueva respiración parcial y en la anestesia de bajo flujo, capnografía facilita el ajuste de flujo de gas 


\section{La capnografía: estudio fundamental en el monitoreo del paciente anestesiado}

Vol. 3, núm. 3., (2019)

Marcos Alfredo Romero Pérez; Carlos Brunos Guerrero; Nina Paulette Arteaga Vélez

fresco, que es un determinante importante de los niveles $\mathrm{CO} 2$, ya que puede aumentar la ventilación minuto". (p. 707)

Martínez (2012) acota con relación a la importancia de la capnografía que son variados los estudios que han demostrado ser un método de mayor efectividad para detectar cambios en la respiración. En un estudio para comparar la efectividad de la capnografía contra la pulsioximetría, de una serie de 634 pacientes postoperados, los cuales se encontraban recibiendo analgesia controlada por el paciente (PCA), "se detectaron 9 casos con bradipnea $<6$ respiraciones por minuto, todos mediante capnografía no así con pulsioximetría. Una detección temprana de la obstrucción de la vía aérea, depresión respiratoria y apnea, podrían prevenir la hipoxia e hipercarbia evitando complicaciones". (p. S20)

No obstante, la eficacia de la técnica usada para la monitorización de la respiración en pacientes bajo anestesia, depende en gran medida del tipo de situación que se presente, por ejemplo, para la detección de la intubación selectiva, la pulsioximetría es el método con mayor eficacia. En virtud de lo cual, lo más recomendable es que ambos estudios se complementen, sobre todo durante la intubación.

\section{Conclusiones.}

El uso de guías de prácticas clínicas para el monitoreo anestésico se encuentra relacionado con una reducción importante de las tasas de complicaciones y mortalidad. El cumplimiento de las normas y estándares básicos contenidos en estas guías garantizan una mayor seguridad al paciente sometido a la anestesia. 


\section{La capnografía: estudio fundamental en el monitoreo del paciente anestesiado}

Vol. 3, núm. 3., (2019)

Marcos Alfredo Romero Pérez; Carlos Brunos Guerrero; Nina Paulette Arteaga Vélez

La capnografía constituye el estándar de oro de la seguridad del paciente bajo anestesia y se encuentra en un nivel altamente recomendado en todas las guías de prácticas clínicas para procedimientos que involucren anestésicos. Su uso conjunto con otros estudios de monitorización tales como la oximetría de pulso, la electrocardiografía o la presión arterial, aporta gran información sobre el estado metabólico, hemodinámico y respiratorio del paciente, y permite identificar de forma más temprana cualquier anomalía clínica que aparezca.

\section{Bibliografía.}

Álvarez, G., \& Ochoa, G. (2013). Monitoreo anestésico básico. Revista Mexicana de Anestesiología, 36(1), 95-100. Recuperado el 15 de Septiembre de 2019, de https://www.medigraphic.com/pdfs/rma/cma-2013/cmas131r.pdf

Barrado, L., Barroso, S., Patón, G., \& Sánchez, J. (2013). Capnografía, la evolución en la monitorización del paciente crítico. Zona TES(1), 16-23. Recuperado el 16 de Septiembre de 2019, de http://media.zonates.com/02-01/PDF/7-Capnografia.pdf

Dell'Osa, A. H., \& Bernia, J. (Octubre de 2014). Solución de la problemática del cableado para centrales de monitoreo. Universidad Favaloro, FICEN, Buenos Aires, Argentina , 1-4. Recuperado el 16 de Septiembre de 2019, de https://www.researchgate.net/publication/273761410_Solucion_de_la_problematica_del_ cableado_para_centrales_de_monitoreo_multiparametrico

Hernández Cortez, E. (Agosto de 2017). Seguridad en Anestesiología. Anestesia en México, 29(2), 1-2. Recuperado el 15 de Septiembre de 2019, de http://www.scielo.org.mx/pdf/am/v29n2/2448-8771-am-29-02-00001.pdf

Instituto Nacional del Cáncer de los Estados Unidos. (20 de Mayo de 2019). Diccionario de cáncer, Diccionario de Cáncer. Recuperado el 10 de Septiembre de 2019, de https://www.cancer.gov/espanol/publicaciones/diccionario/def/anestesia

Martínez, H. (2012). Avances en el uso de la capnografía en pacientes no intubados durante anestesia. Revista Mexicana de Anestesiología, 35(1), S19-S21. Recuperado el 16 de Septiembre de 2019, de https://www.medigraphic.com/pdfs/rma/cma2012/cmas121a5.pdf

Mille, J., Aréchiga, G., \& Portela, J. M. (2011). Estándares internacionales para la práctica segura de la anestesiología. Revista Mexicana de Anestesiología, 34(1), S84-S87. 


\section{La capnografía: estudio fundamental en el monitoreo del paciente anestesiado}

Vol. 3, núm. 3., (2019)

Marcos Alfredo Romero Pérez; Carlos Brunos Guerrero; Nina Paulette Arteaga Vélez

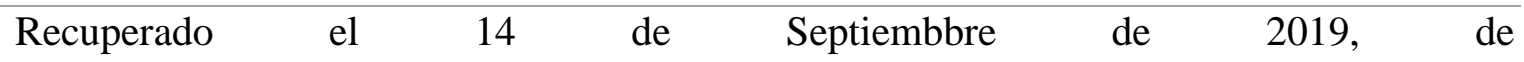
https://www.medigraphic.com/pdfs/rma/cma-2011/cmas111u.pdf

Morales Carbonell, M. A. (2014). "Uso de la capnografía en urgencias. Curso Experto Universitario en Atención al Trauma Grave (2012/13), Universidad Internacional de Andalucía , Andalucía. Recuperado el 16 de Septiembre de 2019, de https://dspace.unia.es/bitstream/handle/10334/3425/0610_Morales.pdf?sequence=3

Navarrete, V. (2011). Medición del co2 espirado. Capnografía Anestesiología, 1-8. Recuperado el 15 de Septiembre de 2019, de http://files.sld.cu/anestesiologia/files/2011/05/mediciondel-co2-espirado.pdf

Organización Mundial de la Salud. (20 de Marzo de 2015). Fortalecimiento de la atención quirúrgica esencial y de emergencia, y de la anestesia, como componentes de la cobertura sanitaria universal. Recuperado el 15 de Septiembre de 2019, de http://apps.who.int/gb/ebwha/pdf_files/WHA68/A68_31-sp.pdf

Ortega, J. (2013). Monitoreo respiratorio. Capnografía. Revista Mexicana de Anestesiología, 36(1), S101-S103. Recuperado el 17 de Septiembre de 2019, de https://www.medigraphic.com/pdfs/rma/cma-2013/cmas131s.pdf

Pérez de Mendiguren, P. (2017). Guía rápida y póster sobre capnografía. Trabajo de Fin de Grado, Universidad Pública de Navarra, Navarra. Recuperado el 14 de Septiembre de 2019, de https://academicae.unavarra.es/bitstream/handle/2454/26688/TFG\%20Paula\%20P\%C3\%A9rez\%20de\%20 Mendiguren.pdf? sequence $=2 \&$ is Allowed $=y$

Romero, M., Guerrero, C., \& Arteaga, N. (2018). Importancia de la capnografía durante el monitoreo anestésico. RECIAMUC, 3(2), 688-711. Recuperado el 16 de Septiembre de 2019, de http://reciamuc.com/index.php/RECIAMUC/article/view/362/375 